\title{
El Artículo 170 de la Constitución Suiza: ¿Qué se ha logrado y qué queda por hacer? ${ }^{1}$
}

Sumario : I. Introducción. II. Sentido y alcance del artículo 170 CS. III. ¿Qué se ha logrado? IV. ¿Qué queda por hacer? V. Observaciones conclusivas.

VI. Bibliografía

Resumen: El artículo 170 es una nueva disposición que se ha introducido en el marco de la revisión total de la Constitución. Este artículo no sólo comprende un control de la eficacia de las medidas políticas, sino su determinación y valoración. Se presenta así la duda acerca de qué consecuencias ha tenido la inserción del artículo 170, pero también qué puede esperarse en general del desarrollo de las evaluaciones.

Abstract: Article 170 of the Swiss Constitution is a new disposition which was introduced within the total revision of the Constitution. The abovementioned article comprehends not only a control of the effectiveness of political measures, but also its determination and evaluation. This study addresses the question about the consequences that article 170 has produced and additionally what can be expected in general of the development of evaluations.

\section{I. introducción}

El artículo 170 de la Constitución de la Confederación Suiza (CS) encomienda al parlamento velar "que se evalúe la eficacia de las medidas tomadas por la Confederación".

Esta disposición es nueva y ha sido introducida por vez primera en 1999 en el marco de la revisión total de la Constitución. Esto se debe en buena medida a la iniciativa y propuestas de las Comisiones de Expertos instauradas por las Comisiones de Política Estatal de la Asamblea Federal que, particularmente en la figura de Pierre Moor, se han pronunciado a favor de la creación de un órgano independiente para la evaluación de las medidas tomadas por el Estado.

¿Cuál ha sido el efecto de esta disposición? ¿Se ha cumplido esta encomienda al Parlamento? ¿Qué tan amplio ha sido, en todo caso, su cumplimiento? ¿Qué queda por hacer?

\footnotetext{
${ }^{1}$ Traducción del alemán de Mauro Arturo Rivera. Este artículo fue originalmente publicado como "Artikel 170 der Bundesverfassung: Was wurde erreicht, was ist noch zu tun?", Gesetzgebung \& Evaluation, 2005/1, Berna.

${ }^{2}$ Luzius Mader es doctor en Derecho por la Universidad de Ginebra. Actualmente es Director Adjunto de la Oficina Federal de Justicia en Berna, donde dirige la División de Derecho Público y es profesor asociado en el Institut de hautes études en administration publique (IDHEAP) de la facultad de derecho de la Universidad de Lausana.
} 
Intentaré brindar elementos para responder a esta pregunta. Previamente, me gustaría realizar ciertas observaciones sobre el sentido y alcance de la disposición constitucional, cuyo texto no necesariamente expresa lo que en realidad quiere decir.

\section{Sentido y alcance del Artículo 170 CS}

El sentido y alcance del artículo $170 \mathrm{CS}$ ha sido con mayor o menor detalle discutido en distintas publicaciones acerca de la nueva Constitución federal. Remito sobre todo a los comentarios de Charles-Albert MORAND ${ }^{3}$, Philippe MASTRONARDI $^{4}$, Jean-François AUBERT ${ }^{5}$ y Thomas SÄGESSER ${ }^{6}$. Me gustaría poner sobre relieve cuatro puntos:

- En primer lugar, me parece importante señalar que aún cuando ciertamente el artículo 170 CS encomienda una tarea al Parlamento, éste no es el único actor al que tal encomienda concierne ni se le ha asignado al Parlamento una responsabilidad absoluta o competencia exclusiva sobre el control de la eficacia de las medidas tomadas por el estado. También el gobierno y la Administración Pública tienen esta responsabilidad y competencia. El parlamento debe, sin embargo, cuidar que tanto Gobierno como Administración cumplan efectivamente con este mandato y por consiguiente cumplan su responsabilidad.

- En segundo lugar, el artículo 170 CS emplea conscientemente la noción amplia de "Medidas de la Confederación". Este concepto incluye todo tipo de acción, toda actividad de los órganos de la federación y de órganos que ejecutan o llevan a cabo medidas de la Federación. Ello implica también a autoridades cantonales o particulares a quienes se les haya confiado el desempeño de tareas de la federación. La forma jurídica de tales medidas (¿legislación, decisiones concretas, actos materiales administrativos?) es aquí irrelevante. El artículo 170 constitucional no basa su redacción en categorías dogmáticas jurídicas, sino que alude a una designación integral muy general de las medidas tomadas por el Estado, cuyo foco se dirige evidentemente al nivel federal.

- En tercer lugar, el artículo 170 CS no sólo se refiere al control posterior. La dimensión prospectiva también se encuentra comprendida aún

3 Morand, Charles Albert, "L'évaluation des effects des mesures étatiques", en Thürer, Daniel; Aubert, Jean-François y Müller, Jörg Paul (eds.), Droit constitutionnel suisse, Schulthess, Zurich, 2001, págs. 1119-1129.

4 Mastronardi, Philippe, "Kommentar zu Art. 170", en Ehrenzeller, Bernhard; Mastronardi, Philippe; Schweizer, Rainer J. y Vallender, Klaus A. (eds.), Die schweizerische Bundesverfassung, Schulthess, Zurich, 2002, págs. 1677-1682.

5 Aubert, Jean-François y Mahon, Pascal, Petit commentaire de la Constitution fédérale de la Confédération suisse du 18 avril 1999, Zürich/Basel/Genf, 2003, págs. 1289 y ss.

6 Sägesser, Thomas, "Kommentar zu Art. 170, en Sägesser, Thomas (ed.), Die Bundesbehörden, Berna, Stäpfli, págs 345-353. 
cuando la palabra control (Überprüfung) bajo estas circunstancias podría arrojarnos hacia otra dirección. Basta aquí con consultar la redacción francesa en que se utiliza la palabra "évaluation". Las evaluaciones pueden -y deben- efectuarse tanto de forma prospectiva, es decir, con vista a medidas y decisiones estatales aún por tomarse, como de forma retrospectiva, es decir, después de que una decisión o medida haya sido adoptada. Las dos perspectivas son complementarias. Los análisis de impacto prospectivo facilitan el control retrospectivo; y en sentido contrario, el control retrospectivo nos aporta indicios y fundamentos para evaluaciones prospectivas posteriores.

- En cuarto y último lugar, en contra de la redacción textual del artículo, no sólo se plantea la duda acerca de la eficacia, es decir, la medida o grado en que se ha cumplido el objetivo de las medidas. La eficacia es sólo un aspecto, sólo un criterio de valoración. Por tanto, el control (Überprüfung) en el sentido del artículo 170 CS también se interesa por la cuestión de si una determinada medida ha sido implementada o puede ser implementada de acuerdo al criterio decisorio (efectividad, observación, cumplimiento, aplicación) y si además de la consecución del objetivo normativo se han producido otros efectos (efectos secundarios, efectos tardíos, etc.).

\section{III. ¿Qué se ha logrado?}

Ahora bien, ¿Qué se ha logrado gracias al artículo 170 CS? Evidentemente sería completamente inadecuado reconducir a esta disposición constitucional todo lo que ha sucedido en los últimos cuatro o cinco años en el campo de la Evaluación de Medidas de la Federación. El artículo 170 presenta la naturaleza de ser una culminación constitucional o, en cierto sentido una "corona" constitucional de un desarrollo que ha tenido lugar en las dos décadas previas a la aprobación de la nueva Constitución y de igual forma es un impulso para nuevos esfuerzos de mejora -también para una institucionalización más fuertede la evaluación de la actividad estatal.

En este sentido, es un ejemplo de un desarrollo de lo que en otro contexto ha sido denominado por Charles-Albert MORAND como "inversión normativa", es decir, un desarrollo contrario a la concepción dogmática de la jerarquía normativa de las disposiciones jurídicas; construido en una dirección de abajo hacia arriba en lugar de arriba hacia abajo.

Mucho habría ocurrido aún en ausencia del artículo 170 CS y mucho seguirá aún sin realizarse a pesar de esta disposición.

El desarrollo de la demanda estatal de evaluaciones, la ampliación de las capacidades evaluativas (oferta) y la profesionalización en este campo, indudablemente se habrían llevado a cabo aún en ausencia de este artículo. Tomando un posicionamiento o valoración que Dieter FREIBURG ha hecho ya 
casi exactamente hace cuatro años ${ }^{7}$ : La siembra se habría producido aún sin el artículo 170 CS, la evaluación política habría echado raíces aún en ausencia de esta disposición constitucional y se habría establecido cuantitativa y cualitativamente de cualquier manera a un nivel comparable al de otros países.

No obstante, el artículo 170 CS no es irrelevante. Ha fortalecido o robustecido una tendencia en desarrollo y adicionalmente la ha legitimado; ha despertado la sensibilidad de las autoridades (Parlamento, Gobierno, Administración) por cuestiones relativas al control de la eficacia y la evaluación; y le ha brindado soporte a aspiraciones institucionales en distintas formas y niveles, quizá en parte induciéndolas.

En este orden de ideas, debe mencionarse por un lado la concretización de la disposición constitucional en la nueva Ley del Parlamento y, por otro lado, los trabajos de implementación que se han llevado a cabo por el Gobierno y la Administración Federal en el marco del grupo de contacto interdepartamental "Wirkungsprüfungen" (conocido como IDEKOWI) y que aún hoy en día se siguen produciendo.

Por lo que respecta a la nueva Ley del Parlamento, me gustaría referirme a cinco provisiones, a saber, artículos 141, 26, 27 y 44 párrafo I incisos e y f, así como al artículo 54 párrafo 4 . De igual forma podría mencionarse el artículo 52 , párrafo II que menciona el criterio de los trabajos de auditoría de las Comisiones de Control (Geschäftsprüfungskommissionen). El artículo 141 se refiere a la evaluación prospectiva y juicio de los efectos. Continúa con el artículo 43 de la antigua Ley sobre la relación entre los Consejos (Geschäftsverkehrsgesetz) y asienta el fundamento para la concreción administrativa interna en la forma de la Guía para la redacción de las opiniones y reportes del Consejo Federal (Botschaftleitfaden) y la guía para la presentación de proyectos de reforma constitucional, leyes y reglamentos de la Asamblea Federal (Botschaftsschema ${ }^{8}$ ). En este orden de ideas se encuentran también las Directrices del Consejo Federal para la presentación de las repercusiones económicas de los proyectos de ley de la Federación así como la Check list y el Manual sobre de Evaluación del Impacto Regulatorio.

En el artículo 26 se menciona el criterio de la eficacia -junto a otros- como un criterio explícito para la supervisión parlamentaria.

El artículo 27 hace referencia específica al control de la eficacia. Representa la verdadera concretización del artículo 170 CS a nivel legislativo y establece, entre otras cosas, que el Parlamento puede requerir al Gobierno la realización de evaluaciones o puede llevar a cabo tal tarea por sí mismo.

7 Freiburghaus, Dieter, "Cui bono? Editorial", Boletín no. 14, SEVAL, 2000.

${ }^{8} \mathrm{http}: / / \mathrm{www} . \mathrm{admin} . \mathrm{ch} / \mathrm{ch} / \mathrm{d} / \mathrm{bk} / \mathrm{sprach} /$ internet/bolf/ 
El artículo 44 párrafo 1 (en particular, incisos e y f) precisan las tareas y legitimación de las Comisiones Legislativas en lo que respecta al control de la eficacia. Finalmente, el artículo 54, párrafo 4, asegura la coordinación al hacer la solicitud de las evaluaciones. Otorga a la Conferencia de Coordinación de los Presidentes de la Comisión de Vigilancia y Delegación de Vigilancia una correspondiente competencia de coordinación.

En comparación con la antigua Ley sobre la relación entre los Consejos se ha llevado a cabo un claro fortalecimiento legislativo de las cuestiones relativas a la evaluación en el terreno parlamentario. Considero satisfactorio el hecho de que, mediante la elaboración de las disposiciones correspondientes, se hayan clarificado ciertos conflictos de competencia (positivos, no negativos) entre las Comisiones Legislativas y la Comisión de Vigilancia.

Esto es por lo que respecta al ámbito parlamentario. ¿Cuál es la situación en el Gobierno y la Administración? Para ello debo remitirme especialmente a los trabajos del anteriormente mencionado Grupo de Contacto Interdepartamental "Wirkungsprüfungen" (IDEKOWI). Este grupo se estableció en el año 2002 a través de la Conferencia de Secretarios Generales. El grupo ha sido dirigido por W. BUSSMAN y tenía la encomienda de realizar un informe acerca de la implementación del artículo $170 \mathrm{CS}$ en el Poder Ejecutivo. El informe fue concluido el 14 de junio de 2004 y el 3 de noviembre de 2004 fue hecho del conocimiento del Gobierno. Se les ha encomendado a las oficinas gubernamentales, los Departamentos y la Cancillería Federal aplicar la mayoría de las medidas propuestas. Esto ahora irá de la mano.

Evidentemente no puedo entrar aquí en detalle acerca de las sugerencias y conclusiones de IDEKOWI. En general debe notarse que el informe no propone ninguna innovación fundamental: debe construirse a partir de lo existente, la utilidad práctica de las evaluaciones debe ser mejorada y ciertas carencias deben ser remediadas.

El informe aclara particularmente las tareas y responsabilidad dentro de las evaluaciones de los organismos especializados, especialmente de órganos con una participación transversal en la preparación de la legislación (Ministerio de Justicia, Hacienda Federal, Cancillería Federal), los Departamentos así como el Consejo Federal. A continuación, precisa el trabajo conjunto de la Administración, el Gobierno y el Parlamento. Finalmente, también aboga por la realización de algunos ajustes legales, principalmente en la Ley Orgánica del Gobierno y la Administración y su reglamento respectivamente. Una disposición general en la Ley Orgánica del Gobierno y la Administración podría al menos, en parte, hacer innecesario el uso específico de cláusulas de evaluación. 


\section{IV. ¿Qué queda por hacer?}

Me parece claro que este informe y las medidas propuestas allí contenidas -así como diversas nuevas disposiciones de la Ley del Parlamento- no podrían haber sido desarrolladas sin el artículo 170 de la Constitución Federal. La nueva disposición constitucional, sin duda alguna, ha desatado diversas cuestiones. Aquí habría, naturalmente, mucho que podría mencionarse, acerca de la reorientación y fortalecimiento en el campo del control de finanzas ${ }^{9}$, la adopción de numerosas cláusulas de evaluación específicas ${ }^{10}$, el incremento del trabajo relativo al control de subvenciones ${ }^{11}$ así como la creación de una red administrativa ${ }^{12}$.

Debo, sin embargo, elevar algunas cuestiones que quedan por hacer. Para ello puedo igualmente basarme en el informe presentado por IDEKOWI: La tarea ahora consiste en llevar a cabo las medidas propuestas allí contenidas. Esto requiere, ante todo, decisiones de la Conferencia de Secretarios Generales, del Consejo Federal, en todo caso también del legislador y subsecuentemente requerirá de medidas de aplicación prácticas. No puede quedarse en deseos devotos, declaraciones de intenciones y melódicas disposiciones jurídicas. Las medidas relativas a la evaluación deben ser implementadas en la práctica.

Para que esto suceda, deben cumplirse una serie de precondiciones:

-Debe mantenerse o aún fortalecerse la sensibilidad de las autoridades políticas y administrativas acerca de la problemática de la evaluación; en esto tienen una especial responsabilidad las mencionadas agencias interdepartamentales.

- La oferta debe incrementarse cuantitativa y sobre todo cualitativamente; con ello se plantea una tarea que puede ser también realizada por la Asociación Suiza de Evaluación (SEVAL). Aquí deben recordarse los Estándares de Evaluación ${ }^{13}$ que son elaborados por esta asociación.

- Debe incrementarse la buena voluntad de los políticos para discutir seriamente los resultados de las evaluaciones -y no sólo encomendar su realización-. En este contexto debe darse también una clarificación de los roles para evitar falsas concepciones o expectativas y así evitar igualmente frustraciones innecesarias. Tomar en cuentan seriamente las evaluaciones no implica simplemente implementar las sugerencias de las evaluadores y

\footnotetext{
${ }^{9}$ El primero de marzo de 1995 entró en vigor una reforma al artículo 5 de la Ley Federal sobre Control Financiero (SR 614.0), que posibilita las auditorías de desempeño (performance audits), en consonancia con desarrollos internacionales, principalmente los esfuerzos de la Organización Internacional de las Entidades Fiscalizadoras Superiores. La Auditoría Superior Federal ha ampliado sus actividades en esta área hasta el año 2002 y desde entonces las ha fortalecido lentamente.

${ }^{10} \mathrm{http}: / / \mathrm{www}$. ofj.admin.ch/themen/eval/evklausel-d.htm

${ }^{11} \mathrm{http}: / / \mathrm{www}$. efv.admin.ch/d/finanzen/subven/h_rubrik.php

$12 \mathrm{http}: / / \mathrm{www}$.seval.ch/de/documents/seval_Standards_2001_dt.pdf

${ }^{13} \mathrm{http}: / / \mathrm{www}$. seval.ch/de/documents/seval_Standards_2001_dt.pdf
} 
evaluadores. Éstos últimos no deben tener esta expectativa de la política. Si quienes toman las decisiones políticas hiciesen esto, sencillamente no llevarían a cabo su tarea e incumplirían su responsabilidad; sin embargo, deben fundamentar razonablemente el por qué tales sugerencias no han sido adoptadas.

Se plantea la cuestión de si estamos en un momento favorable para lograr realizar ulteriores progresos en el campo de la evaluación de la eficacia de las medidas estatales, es decir, concretamente, llevar a cabo las recomendaciones de IDEKOWI y crear para ello los necesarios presupuestos.

Para ello debe atenderse al contexto fiscal. ¡Las evaluaciones tienen un costo! Existe el peligro de que los recursos económicos necesarios para su realización no se encuentren disponibles en los próximos años, o por lo menos no en la misma medida.

Será decisivo, por tanto, si persiste la apreciación de que las evaluaciones tienen un costo, o si se logra demostrar que las evaluaciones pueden contribuir decisivamente a un ahorro significativo.

En las presentes circunstancias, me parece que aportar esta prueba es una tarea urgente y desafiante de la "Comunidad Evaluadora", es decir, de las evaluadoras y los evaluadores, así como sus empleadores.

Parto de la suposición de que un sistema político construido en base al consenso, interesado en justificar racionalmente hasta cierto punto las decisiones de ahorro drásticas, debería poder aconsejarse sobre ello. Por lo tanto, definitivamente se deben hacer evaluaciones para ayudar a la toma de decisiones y para justificar estas decisiones. En este sentido, la escasez financiera y una más fuerte conciencia de los costos en la administración no conllevan necesariamente un peligro para la tendencia y aspiraciones al desarrollo en el campo de la evaluación de las medidas estatales; pueden también ser una oportunidad que debe ser utilizada.

\section{Observaciones conclusivas}

Permítaseme concluir con una pequeña observación si se quiere un poco herética.

A principios de esta semana me he topado con un artículo en el Neuen Zürcher Zeitung con el título "De premoniciones a certezas"14. En este artículo, que pretende demostrar por qué la política debe basarse en evaluaciones, Andreas BALTHASAR argumenta que, en muchos casos, las evaluaciones « [convierten] premoniciones en certezas».

14 Balthasar, Andreas (2004), "Von Vorahnungen zu Gewissheiten- Warum die Politik auf Evaluationen angewiesen ist", Neue Zürcher Zeitung, 8 de junio, Zürich. 
Esta afirmación me parece ahora, sin embargo, un poco arriesgada. $Y$ sobre todo: me parece que se encuentra unida al peligro de despertar falsas expectativas que después, a su vez, desatará las correspondientes frustraciones. Contemplado a largo plazo, quizá sea más prometedor brindar una idea realista acerca de las posibilidades de rendimiento de las investigación evaluativa y explicó: «No obstante las promesas dinámicas en la aplicación del proyecto aparato del proceso de investigación, inmediatamente se palpa una realidad que con una abrumadora complejidad se sabe oponer a la impertinencia investigadora. Esto no se opone a la investigación empírica, sino sólo a nociones ingenuas de su utilidad práctica ${ }^{15}$ ».

Las certezas no son necesarias, las plausibilidades bien fundadas bastan 0 más bien brindan un adecuado valor adicional. Las decisiones sobre medidas estatales dependen siempre -abierta o tácitamente- en presuposiciones, en hipótesis de impacto sobre relaciones causales entre las medidas en cuestión y la realidad social. El desarrollo de la evaluación coadyuva a que estas hipótesis no sólo sean explícitas y con ello se conviertan en verificables; también puede asegurarse que esta evaluación -sea ya prospectiva o retrospectiva- se lleve a cabo de un modo metódico, es decir, comprensible. Aún cuando esta evaluación no proporcione certezas permite -en la mayoría de los casos con un esfuerzo razonable-, obtener algo más que estimaciones meramente intuitivas o impresionistas.

\section{Bibliografía}

Aubert, Jean-François y Mahon, Pascal, Petit commentaire de la Constitution fédérale de la Confédération suisse du 18 avril 1999, Zürich/Basel/Genf, 2003, págs. 1289 y ss.

Balthasar, Andreas (2004), "Von Vorahnungen zu Gewissheiten- Warum die Politik auf Evaluationen angewiesen ist", Neue Zürcher Zeitung, 8 de junio, Zürich

Freiburghaus, Dieter, "Cui bono? Editorial”, Boletín no. 14, SEVAL, 2000.

Holänd, Armin, "Von Machen und Messen von Gesetzen- Erkenntnisse aus der Forschungspraxis zur Reichweite der Gesetzesevaluation", Zeitschrift für Rechtssoziologie, H. 2/1989, p. 219.

Mastronardi, Philippe, “Kommentar zu Art. 170”, en Ehrenzeller, Bernhard; ------

Schweizer, Rainer J. y Vallender, Klaus A. (eds.), Die

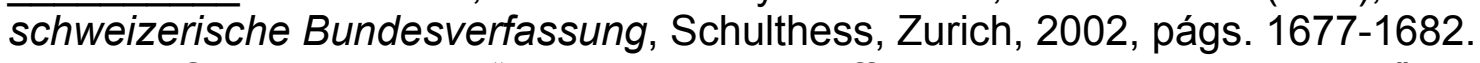
Morand, Charles Albert, "L'évaluation des effects des mesures étatiques", en Thürer, Daniel; Aubert, Jean-François y Müller, Jörg Paul (eds.), Droit constitutionnel suisse, Schulthess, Zurich, 2001, págs. 1119-1129.

Sägesser, Thomas, "Kommentar zu Art. 170, en Sägesser, Thomas (ed.), Die Bundesbehörden, Berna, Stäpfli, págs 345-353.

\footnotetext{
15 Holänd, Armin, "Von Machen und Messen von Gesetzen- Erkenntnisse aus der Forschungspraxis zur Reichweite der Gesetzesevaluation“, Zeitschrift für Rechtssoziologie, H. 2/1989, p. 219.
} 